\title{
(3749) BALAM: A VERY YOUNG MULTIPLE ASTEROID SYSTEM
}

\author{
DAVID VOKROUHLICKÝ \\ Institute of Astronomy, Charles University, V Holešovičkách 2, CZ-18000 Prague 8, Czech Republic, vokrouhl@cesnet.cz \\ Received 2009 September 17; accepted 2009 October 13; published 2009 October 28
}

\begin{abstract}
Binaries and multiple systems among small bodies in the solar system have received wide attention over the past decade. This is because their observations provide a wealth of data otherwise inaccessible for single objects. We use numerical integration to prove that the multiple asteroid system (3749) Balam is very young, in contrast to its previously assumed age of $0.5-1 \mathrm{Gyr}$ related to the formation of the Flora family. This work is enabled by a fortuitous discovery of a paired component to (3749) Balam. We first show that the proximity of the (3749) Balam and 2009 BR60 orbits is not a statistical fluke of otherwise quasi-uniform distribution. Numerical integrations then strengthen the case and allow us to prove that 2009 BR60 separated from the Balam system less than a million years ago. This is the first time the age of a binary asteroid can be estimated with such accuracy.
\end{abstract}

Key words: minor planets, asteroids

\section{INTRODUCTION}

Decadal asteroid reviews published by the University of Arizona Press mapped the route of binary asteroids from hypothetical to practical, and very useful, objects for planetary science (e.g., Weidenschilling et al. 1989; Merline et al. 2002a). Today, these systems are in the forefront of both observational and theoretical efforts because they provide a number of otherwise inaccessible physical data about asteroids (e.g., Merline et al. 2002a; Richardson \& Walsh 2006; Pravec \& Harris 2007). However, as for single asteroids, only some information is available from direct observations while some other information remains hidden to them. Perhaps the most interesting of such additional data is the age of the binary system. This is because its knowledge can, with properly modeled long-term evolution of the binary or multiple system, constrain tidal parameters of one or several components.

So far we could have only estimated binary-system age from two methods, each of which is largely uncertain or model dependent: (1) direct spacecraft imaging and analysis of the cratering record, such as in the case of (253) Ida (e.g., Greenberg et al. 1996), and (2) binary membership in a dated asteroid family, such as in the case of (283) Emma (e.g., Marchis et al. 2008a).

Here, we introduce a new technique for binary age determination applicable to very young systems and use it in the case of (3749) Balam (Sections 2 and 3; discovery circumstances for this triple system are in Merline et al. 2002b and Marchis et al. 2008b). Moreover, forthcoming all-sky-survey observations will have the power to discriminate between different formation mechanisms of the Balam multiple system (Section 4).

\section{2009 BR60: A PAIRED COMPONENT TO BALAM}

This work directly builds on a recent development of the age determination methods for very young asteroid families (e.g., Nesvorný et al. 2006; Nesvorný \& Vokrouhlický 2006) and single asteroids (e.g., Vokrouhlický \& Nesvorný 2008, 2009; Pravec \& Vokrouhlický 2009). In the second case, the method uses a specific population of asteroids that have twins (or paired objects) on very similar orbits. In both approaches, the age estimation is obtained by direct orbital integration over a sufficiently short period of time, such that growing uncertainty due to chaoticity and/or non-gravitational forces does not invalidate the result.

Fortuitously, the same method can be applied to the Balam case as well. This is because a two-opposition asteroid 2009 BR60 resides on a nearly identical orbit to (3749) Balam and constitutes thus a second component in this pair. Using the tools developed for the orbit analysis of asteroid pairs (Vokrouhlický \& Nesvorný 2008; Pravec \& Vokrouhlický 2009), we note that a mutual distance between (3749) Balam and 2009 BR60 in the five-dimensional space of osculating elements ranges between 3.5 and $4.1 \mathrm{~m} \mathrm{~s}^{-1}$ (see discussion in Pravec \& Vokrouhlický 2009). Checking uniformity of the orbit distribution in the Balam vicinity, we may use the method of Pravec \& Vokrouhlický (2009, Section 4) to show that there is less than $1 \%$ chance that Balam's and 2009 BR60's proximity is a random distribution fluke. Backward integration of the nominal orbits for the two asteroids, with gravitational perturbations of all planets, also indicates convergence of secular angles some 200-300 kyr ago. Finally, Pravec \& Vokrouhlický (2009) used proximity of the asteroid orbits in the proper-element space as an independent tool to justify existence of the asteroid pairs. We thus created a limited number of clones, 250 for each of the two asteroids, sampling the uncertainty ellipsoid in the osculating orbital space and propagated their orbits for $5 \mathrm{Myr}$ with all planetary perturbations included. The output was analyzed and synthetic proper elements, compatible with those of Knežević et al. (2002), were computed. With random assignment of the orbits in pairs, we computed their distance in the proper-element space. Distances obtained using this method have a quasi-Gaussian distribution with a mean value of $\sim 9 \mathrm{~m} \mathrm{~s}^{-1}$ and a standard deviation $\sim 4 \mathrm{~m} \mathrm{~s}^{-1}$ (there is also a component showing larger proper-element distances, but detailed scrutiny shows these are orbits affected by the nearby $9 / 16$ mean motion resonance with Mars, additionally to the long-term effects of the $z_{2}$ secular resonance). This small mean distance of proper elements of (3749) Balam and 2009 BR60 is comparable to the escape velocity from the larger component $\left(3.5-5 \mathrm{~m} \mathrm{~s}^{-1}\right)$.

All these independent lines of evidence show that (3749) Balam and 2009 BR60 represent a statistically robust pair of asteroids with a common origin within the past half to $1 \mathrm{Myr}$. Table 1 summarizes orbital information about the two asteroids 
Table 1

Osculating Orbital Elements, their Uncertainties and Other Parameters of the Asteroid Pair (3749) Balam and 2009 BR60

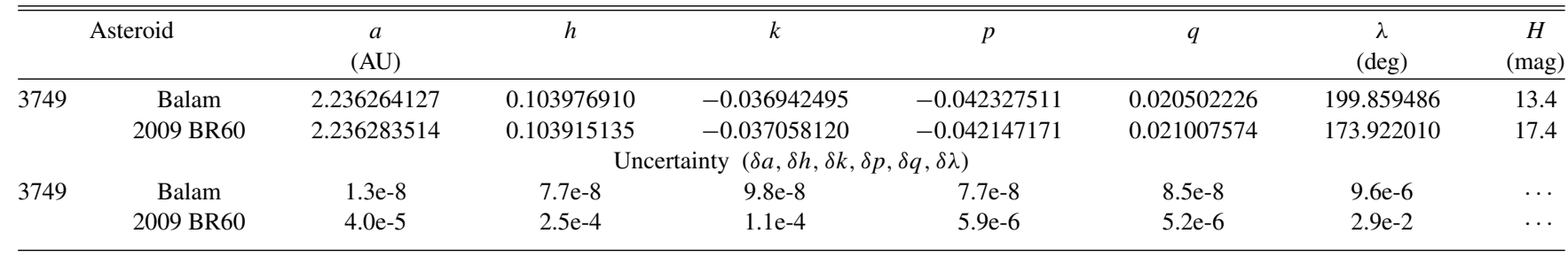

Notes. Equinoctial orbital elements of the Balam pair members as of MJD 55000.0: $a$ is semimajor axis, $(h, k)=e(\sin \varpi, \cos \varpi)$ where $e$ is the eccentricity and $\varpi$ is the longitude of perihelion, $(p, q)=\tan (i / 2)(\sin \Omega, \cos \Omega)$ where $i$ is the inclination and $\Omega$ is the longitude of node, and $\lambda=\varpi+M$ is the mean longitude in orbit ( $M$ is the mean anomaly). Default reference system is that of mean ecliptic J2000. Orbital solution, together with formal standard deviation uncertainties, is from the AstDyS catalog as of 2009 September (e.g., Knežević et al. 2002). The adopted absolute magnitude values $H$ are from MPC. In general, their uncertainty is \pm 0.5 mag, but it may be smaller for (3749) Balam because of its small light-curve amplitude (e.g., Marchis et al. 2008b).

as of 2009 September. While the orbit of (3749) Balam is fairly well constrained, that of 2009 BR60 is rather poor reflecting only a small number of astrometric observations over just two oppositions (see Section 4). Still, existence of this asteroid is fundamental for our work and we seek to understand its implications.

\section{AGE CONSTRAINT FOR THE BALAM SYSTEM}

While indicative, the backward propagation of the nominal orbits is not sufficient for the age determination. This is because any orbit initially located in the present-time uncertainty ellipsoid is statistically equivalent. Those orbits are confined into a small volume in the orbital element space now (e.g., Table 1) but typically quickly diverge as time proceeds into the future or past. As a result, we must include many of these orbits into our search for the Balam system age; we call these orbits geometric clones. Additionally, orbital history of small asteroids is strongly affected by the thermal (Yarkovsky) forces (e.g., Bottke et al. 2006). Any of the geometric clones described above may thus have a spectrum of variants, depending on the strength and sign of Yarkovsky forces which are presently unconstrained for both (3749) Balam and 2009 BR60. These variants are called the Yarkovsky clones. Given the tighter confinement and larger size of (3749) Balam, we need fewer geometric and Yarkovsky clones for this asteroid.

With these preliminary comments, we can now describe our main simulation and analysis. We used 21 geometric and 31 Yarkovsky clones for (3749) Balam, and 61 geometric and 71 Yarkovsky clones for 2009 BR60, altogether near to 5000 bodies. Their initial conditions together with planetary initial conditions were taken at MJD 55000.0 and propagated backward over 1 Myr time span. We used SWIFT_MVS integrator with a time step of $5 \mathrm{~d}$, modified to include effects of the thermal (nonconservative) forces. We stored positions and velocities of all integrated variants for both asteroids every $50 \mathrm{yr}$ (output sampling). At each output, we performed a statistical analysis of the orbital proximity between the two clouds of clones for (3749) Balam and 2009 BR60. To proceed quantitatively, we constructed 5 million random pair identifications and evaluated two versions of a target function (for more details see Nesvorný \& Vokrouhlický 2006; Vokrouhlický \& Nesvorný 2008, 2009):

1. $\Delta V^{\prime}=n a \sqrt{(\sin i \Delta \Omega)^{2}+0.5(e \Delta \varpi)^{2}}$, where $n$ is mean motion, $a$ is semimajor axis, $e$ is eccentricity, $i$ is inclination for Balam's orbit clone, and $\Delta \Omega$ and $\Delta \varpi$ are differences of longitude of node and pericenter for clones in the tested pair, and

\section{2. $\Delta R$ being the true distance of the two clones in space.}

The first option, $\Delta V^{\prime}$, monitors convergence of secular angles $\Omega$ and $\varpi$ disregarding other orbital elements $(a, e, i)$, which are close enough even for the current orbital elements. For instance, the current orbital difference value of (3749) Balam and 2009 BR60 in the semimajor axis is $\sim 2 \times 10^{-5}$ AU, which translates to a velocity difference of $\sim 0.2 \mathrm{~m} \mathrm{~s}^{-1}$ only. However, the current difference of $\sim 0.7$ in $\Omega$ and $\varpi$ translates into a velocity difference of $\sim 35 \mathrm{~m} \mathrm{~s}^{-1}$. So this method, originally used for reconstruction of very young asteroid families, seeks orbital confinement below a given threshold in the fastest diverging orbital elements only.

The second target function, the true distance $\Delta R$ of clones in space, is a more difficult and ambitious test. Obviously, many clones are far away from each other, so that we restrict ourselves to evaluate a minimum value of $\Delta R$ over randomly sampled clones as an expression of theoretical limit of proximity of the two asteroids in the past. We also evaluate relative velocity $\Delta V$ of the close clones, because only low-velocity encounters are compatible with formation scenarios mentioned in Section 4.

The upper panel of Figure 1 shows the normalized distribution of number of trials in our numerical experiment that provided $\Delta V^{\prime}$ value smaller than 2 and $5 \mathrm{~m} \mathrm{~s}^{-1}$, comparable or smaller than the escape velocity from (3749) Balam. The bottom panel of the same figure gives one example of many similar solutions where a good convergence of the secular angles-longitude of node and pericenter-was achieved. At the convergence of $\Omega$ and $\varpi$, the semimajor axis, eccentricity, and inclination of the two clones all have differences smaller than $\sim 2 \times 10^{-5}$ despite their much larger differences in the intermediate instants. At the peak of the distribution shown in the top panel of Figure 1, some $150 \mathrm{kyr}$ ago, number of pairs of clones with $\Delta V^{\prime} \leqslant 5 \mathrm{~m} \mathrm{~s}^{-1}$ reached up to $5 \%$ of all trials.

Figure 2 shows results of the strengthened test where the true Cartesian state vectors, distance in space $\Delta R$ and relative velocity $\Delta V$, of pairs of clones for (3749) Balam and 2009 BR60 were computed. The distribution in the upper panel shows the number of cases that led to a close and low-velocity encounter; in quantitative terms we set the distance $\Delta R$ limit to two and three times radius $R_{\text {Hill }}$ of Balam's gravitational influence $\left(R_{\text {Hill }} \simeq 1200 \mathrm{~km}\right)$ and the mutual velocity to be smaller than 2 and $5 \mathrm{~m} \mathrm{~s}^{-1}$ (about the escape velocity from Balam). At the peak of the distribution, we recorded about 10 matches from 5 million trials at each output. The middle panel of Figure 2 shows the minimum distance $\Delta R$ between two clones at each output, and the bottom panel of the same figure shows the relative velocity $\Delta V$ of the clones at the minimum distance. 


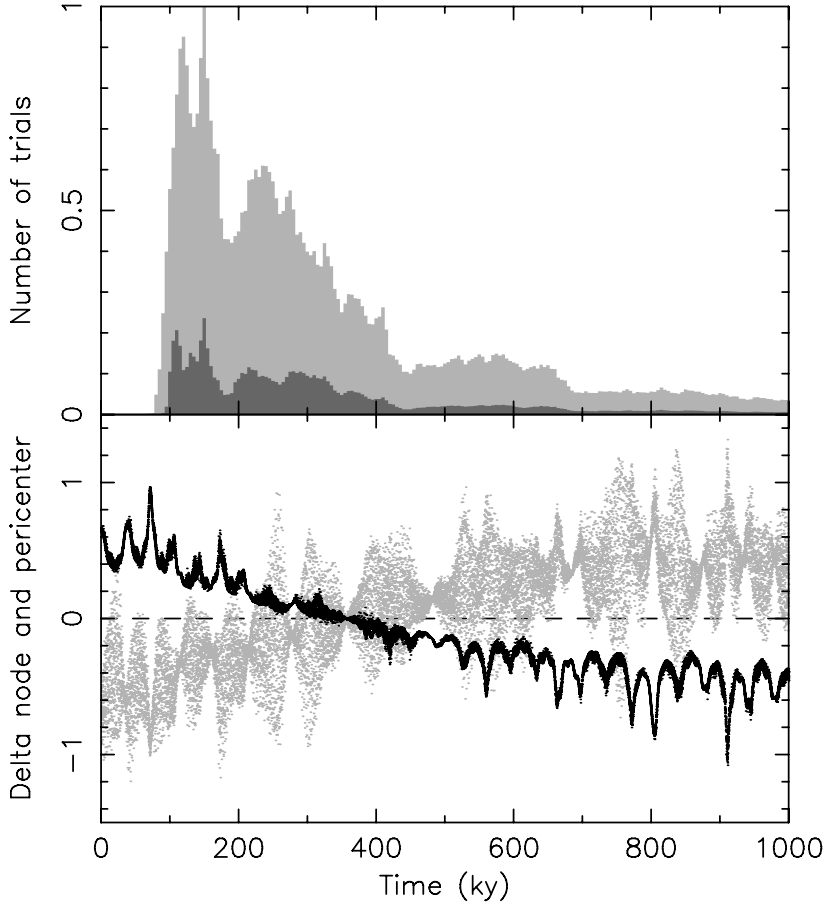

Figure 1. Top: number of trials that provided $\Delta V^{\prime} \leqslant 5 \mathrm{~m} \mathrm{~s}^{-1}$ (light gray) and $\Delta V^{\prime} \leqslant 2 \mathrm{~m} \mathrm{~s}^{-1}$ (dark gray) binned into $5 \mathrm{kyr}$ intervals. The ordinate is normalized to maximum of the former distribution. Bottom: an example of a good convergence of secular angles of (3749) Balam and 2009 BR60 in the past. Black is the difference $\Delta \Omega$ in longitude of node and gray is the difference $\Delta \omega$ in argument of pericenter (both referred to Balam). At $\sim 350 \mathrm{kyr}$, we have $\Delta V^{\prime} \sim 0.4 \mathrm{~m} \mathrm{~s}^{-1}$

Between 200 and $500 \mathrm{kyr}$ ago, the closest approaches reach the limit of $R_{\text {Hill }}$ (the slight offset by a factor of 2-3 is likely due to simplifications in the modeling of the Yarkovsky forces; see Nesvorný \& Vokrouhlický 2006; Vokrouhlický \& Nesvorný 2008). The typical minimum relative velocities are of the order $1 \mathrm{~m} \mathrm{~s}^{-1}$ or less, implying a very gentle separation of 2009 BR60 from the Balam system in the past (these values are an order of magnitude smaller than the escape velocity from (3749) Balam; compare with a similar solution in Vokrouhlický \& Nesvorný 2009).

Combining information from our two approaches above, Figures 1 and 2, we conclude that the age of the Balam's system ranges from 150 to $500 \mathrm{kyr}$. A tail of possible solutions for larger values of age represents less than $10 \%$ of all cases.

\section{DISCUSSION AND IMPLICATIONS}

Results from our analysis will be significantly improved when accurate astrometry of 2009 BR60 is obtained during its next opposition in 2010 June and August. We estimate that the uncertainties in the orbital elements of this asteroid (Table 1) may shrink by a factor of $\sim 50-100$ and that will help to reduce the uncertainty in the age determination. While somewhat south on the sky, these observations should not be difficult for regular instrumentation of the survey stations. Nevertheless, we prompt observers to take advantage of this opportunity.

Turning now to the implications of our solution, we note that the young age of the Balam system helps to better understand high eccentricity of the resolved satellite orbit $(\sim 0.9$; see comments in Marchis et al. 2008a, Section 5) and a somewhat unusual make-up of this triple system in general. Note that, unlike in the case of other known triple systems, the outer satellite of (3749) Balam is located at a distance equivalent to

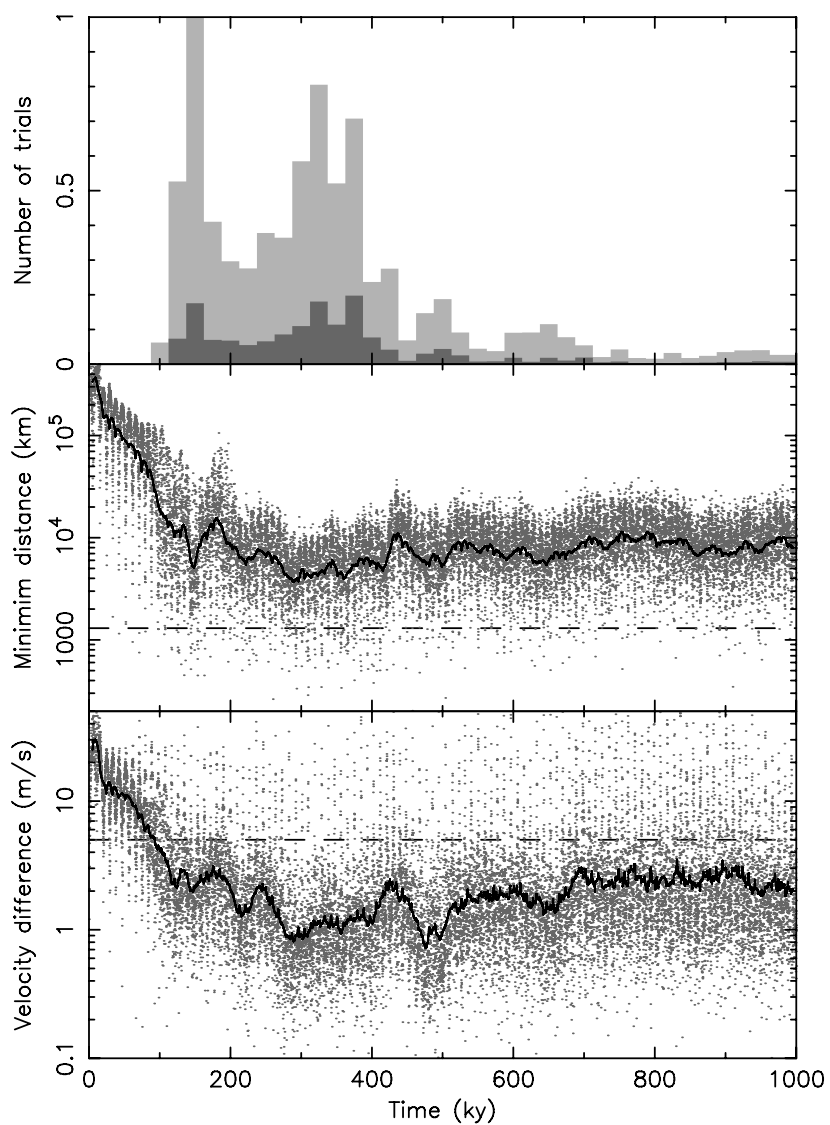

Figure 2. Top: number of trials, binned into $25 \mathrm{kyr}$ intervals, that provided $\Delta R \leqslant 3 R_{\text {Hill }}$ and simultaneously relative velocity $\Delta V \leqslant 5 \mathrm{~m} \mathrm{~s}^{-1}$ (light gray), and $\Delta R \leqslant 2 R_{\text {Hill }}$ and simultaneously relative velocity $\Delta V \leqslant 2 \mathrm{~m} \mathrm{~s}^{-1}$ (dark gray). The ordinate is normalized to maximum of the former distribution. Middle: minimum distance $\Delta R$ over 5 million identification trials between Balam and 2009 BR60 clones at each output from our simulation (gray symbols); mean value over $10 \mathrm{kyr}$ running window in black. The dashed line shows estimated Balam's Hill radius for reference. Bottom: relative velocity $\Delta V$ of the clones at the moment of minimum-distance close approaches from the middle panel (gray symbols); mean value over $10 \mathrm{kyr}$ running window in black. The dashed line shows $5 \mathrm{~m} \mathrm{~s}^{-1}$ limit, roughly the estimated escape velocity from Balam, for reference.

a fair fraction of $R_{\text {Hill }}$ (e.g., Marchis et al. 2008a). Next, we are directed to considerations of the Balam system's origin. There are three possibilities:

1. (3749) Balam is the largest fragment of its own asteroid mini-family (see Nesvorný et al. 2006; Nesvorný \& Vokrouhlický 2006). Durda et al. (2004) show that the size ratio $\sim 5$ between the largest fragment and its satellite is not infrequent in numerical simulations of the asteroid fragmentation.

2. The precursor of (3749) Balam might have undergone an unusual fission process that created the paired component 2009 BR60 in the same way as hypothesized for other asteroid pairs (e.g., Vokrouhlický \& Nesvorný 2008; Pravec \& Vokrouhlický 2009), but parking also some fragments onto bound, satellite orbits.

3. The precursor of (3749) Balam might have been a triple or quadruple system that recently underwent instability through close encounter, or even collision, of its satellites; this process might have preserved the inner satellite but moved fragments of another satellite(s) onto a loosely bound, high-eccentricity orbit and ejected (2009 BR60) from the system. 
The very short rotation period of (3749) Balam, reported by Marchis et al. (2008b), and the size ratio between (3749) Balam and 2009 BR60 may support options 2 and 3 (see, e.g., Scheeres 2007), but they do not contradict option 1. At this moment we are not able to discriminate between all three possibilities given above. The upcoming sky-survey observations of PanSTARRS and LSST should have the power to do so (e.g., Jedicke et al. 2007; Ivezić et al. 2007). If the first possibility is correct, a number of so far unseen family members in the $0.5-1 \mathrm{~km}$ size range should be discovered in the forthcoming years. If no such bodies are found, the second and third options would become more viable formation processes of the Balam system. Moreover, these new-generation data may help us to uncover more twin components on heliocentric orbits to recently formed binary or multiple asteroids. A search with the currently available catalogs and known population of small main-belt binaries did not reveal any further cases than that of Balam.

This work was supported by grants GAČR 205/08/0064 and 205/09/1107 of the Czech grant agency and by the Research Program MSM0021620860 of the Ministry of Education. I also thank Franck Marchis and Petr Pravec for comments on the first version of this Letter.

\section{REFERENCES}

Bottke, W. F., Vokrouhlický, D., Rubincam, D. P., \& Nesvorný, D. 2006, Ann. Rev. Earth Planet. Sci., 34, 157
Durda, D. D., Bottke, W. F., Encke, B. L., Merline, W. J., Asphaug, E., Richardson, D. C., \& Leinhardt, Z. M. 2004, Icarus, 170, 243

Greenberg, R., Nolan, M. C., Bottke, W. F., Petit, J. -M., Geissler, P., \& Durda, D. D. 1996, Icarus, 120, 106

Ivezić, Ž., et al. 2007, in Near Earth Objects, LSST: Comprehensive NEO Detection, Characterization, and Orbits, Our Celestial Neighbors: Opportunity and Risk, ed. A. Milani, G. B. Valsecchi, \& D. Vokrouhlický (Cambridge: Cambridge Univ. Press), 353

Jedicke, R., Magnier, E. A., Kaiser, N., \& Chambers, K. C. 2007, in Near Earth Objects, The Next Decade of Solar System Discovery with PanSTARRS, Our Celestial Neighbors: Opportunity And Risk, ed. A. Milani, G. B. Valsecchi, \& D. Vokrouhlický (Cambridge: Cambridge Univ. Press), 341

Knežević, Z., Lemaitre, A., \& Milani, A. 2002, in Asteroids III, The Determination of Asteroid Proper Elements, ed. W. F. Bottke et al. (Tuscon, AZ: Arizona Univ. Press), 603

Marchis, F., et al. 2008a, Icarus, 195, 295

Marchis, F., et al. 2008b, IAU Circ., 8928, 4

Merline, W. J., Weidenschilling, S. J., Durda, D. D., Margot, J.-L., Pravec, P., \& Storrs, A. D. 2002a, in Asteroids III, Asteroids do Have Satellites, ed. W. F. Bottke et al. (Tuscon, AZ: Arizona Univ. Press), 289

Merline, W. J., et al. 2002b, IAU Circ., 7827, 2

Nesvorný, D., \& Vokrouhlický, D. 2006, AJ, 132, 1950

Nesvorný, D., Vokrouhlický, D., \& Bottke, W. F. 2006, Science, 312, 1490

Pravec, P., \& Harris, A. W. 2007, Icarus, 190, 250

Pravec, P., \& Vokrouhlický, D. 2009, Icarus, in press

Richardson, D. C., \& Walsh, K. J. 2006, Annu. Rev. Earth Planet. Sci., 34, 47

Scheeres, D. J. 2007, Icarus, 189, 370

Vokrouhlický, D., \& Nesvorný, D. 2008, AJ, 136, 280

Vokrouhlický, D., \& Nesvorný, D. 2009, AJ, 137, 111

Weidenschilling, S. J., Paolicchi, P., \& Zappalà, V. 1989, in Asteroids II, Do Asteroids Have Satellites?, ed. M. S. Matthews et al. (Tuscon, AZ: Arizona Univ. Press), 643 